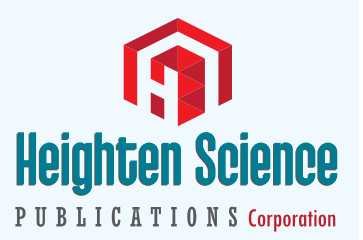

ISSN

2640-284X
*Address for Correspondence: Luisetto M, Applied Pharmacologist, European Specialist laboratory Medicine, Independent Researcher, Italy 29121, Email: Maurolu65@gmail.com

Submitted: 28 February 2018

Approved: 08 March 2018

Published: 09 March 2018

Copyright: @ 2018 Luisetto M, et al. This is an open access article distributed under the Creative Commons Attribution License, which permits unrestricted use, distribution, and reproduction in any medium, provided the original work is properly cited

Keywords: Metabolic-endocrine disease; New pharmacological strategies; Toxicology; Antidotes approach; Kinetics

Check for updates
Review Article

\section{New pharmacological strategies in some metabolic endocrine disorder under a toxicological approach}

\author{
Luisetto $\mathrm{M}^{1 *}$, Ghulam Rasool Mashori ${ }^{2}$ and Cabianca luca ${ }^{3}$ \\ 'Applied Pharmacologist, European Specialist Laboratory Medicine, Independent Researcher, \\ Italy \\ ${ }^{2}$ Department of Medical \& Health Sciences for Woman, Peoples University of Medical and \\ Health Sciences for Women, Pakistan \\ ${ }^{3}$ Biomedical Laboratory, Torino, Italy
}

\section{Abstract}

In this review After Observing biomedical literature (starting from some heart disease) results that some pathological phenomena are deeply involved in some metabolic endocrine condition: Kinetics and gradients in metabolism, catabolism, time related, toxic like effect, electrical cell membrane status, smooth vascular muscle cell hyper-reactivity, platelet iperactivations, central autonomic control after acute stroke, great electrolytes unbalances et other factors as pro-hypertrophic signaling and oxidative stress. Observing actual current therapy in some metabolic endocrine therapy often is used association of drugs (in example in type II diabetes). In Many other pathologies efficacy drug therapy exist, and often only 1 pharmacological molecule resolve the pathological condition. But in many disease even associating 2-3-4 drugs the \% of cure not increase (efficacy, effectiveness). It mean that this drugs strategies are not the really best? Or it mean a low active level? Why for this pathological condition this association drugs in currently use not do the right works as really needed? There is the need for new really efficacy drugs strategy that show a profile of efficacy as requested in order to resolve the pathological condition? Or to be added to the actual therapy? The actual pharmacological strategies in some metabolic endocrine disorder is really the best? Or other strategies can be introduced?

\section{Introduction}

Todayisneeded to introduce newapproach in some metabolic-endocrinepathologies and to do this is useful to Use a toxicological approach, new delivery systems and new diagnostic strategies. (This paper is produced under a pharmaceutical-toxicological approach).

\section{Material and Methods}

Using a review method and observing some literature in biomedical database can be produced a new approach in pharmacological strategies in some relevant metabolicendocrine disease or to prevent it. Observing some works related to some heart disease to try to find strategies that could be translated to the metabolic-endocrine pathology field.

\section{Results}

\section{From literature}

In short communication "Sudden Heart Pathology-a New Research Hipotesys" published in 2017 was reported that "A new method in heart disease clinical staging strategy is needed. New tests that can make possible to stress cardiac metabolism in normal, low and high working conditions or in para-phisio-pathological conditions but in local place (heart situation and not in plasma in example). This new methods can 
be useful in much heart disease and condition to prevent some events. (Heart attacks, ischemic disease, airtime, heart failure, transplants, sports and so on). Every biomedical discipline has specific diagnostic discipline, but it can be useful to translate the various diagnostic system strategies from a discipline to other: this make possible to observe phenomena with a different point of view" [1].

And in "Heartdisease new hypotheses: under endogenous toxicological aspect"work. Was reported that "An endogenous local toxicology aspect time related to verify some pathologic process and phenomena must be considered under a new useful light. In some time related CV local metabolic-catabolic-toxic status are observed some cellular effect resulting in global organ-apparatus failure. The time involved (and kinetics aspect) in resolve some temporary metabolic-catabolic-electric gradients or the global velocity involved in this process can be fundamental to consider. (too rapid evolution or too slow reduction in balancing-equilibrates some physiologic systems)" [2].

According Luisetto M, et al., "In example what happen in SCD in untrained? Why physical training can reduce this event (SCD)? Can be considered a condition of ENDOGENUS toxicity time-KINETICKS related. In this kind of condition is relevant to better observe the time related endogenous intra- toxicity situation involved in some Metabolic- catabolic-electrical cell membrane and other. For example involved in some heart airtime, epileptic status, septic shock and other situation high time related (ischemic coronary spasm etc). In embryology, oncology, toxicology, pathology some heart and brain disease the time is relevant factors added to endogenous local- micro environment and inter- cellular communication (crisis). An intra- local toxicology aspect time related can be considered to better verify some pathologic process under a new light" [3].

And in Surgery and new Pharmacological strategy in some atheroscleroses chronic and acute conditions" in 2017 was reported that "In actual pharmacological therapy some drugs can be added to other medical instruments to improve their activity: in example medicated stent for some coronary disease, or hormonal medical devices used in pregnancy prevention, but other example are known today. In example Carmustine wafer is delivered by delivery systems in some brain cancer and radioactive seed implants in prostatic cancer. Ocular intra virtual implants for some macular degenerations (MABS or cortisones) other implants delivery systems drugs, naltrexone implant for opiate dependence. Other strategies imply carrier use to deliver the drugs in the site of action: In example MABS linked to radioactive isotopes in some relapse of severe Hodgkin disease but many other example in therapy used today. So other chronic conditions can be treated using a combination of drugs with other instrument to improve the clinical outcomes. This to make possible that the ERLICH MUGIC BULLETS can act in the right site reducing the side effect. In example today various medical interventional radiological strategy to treat in coronary and hearth disease with medicate stents positioning or to local use of contrast agents or other valve surgery procedures with global good clinical results" [4]. "Under the light of the article find in this paper but also to other works published a new system "to regenerate" a valve tissue calcified can be considered. Adding 3 strategies (medical interventional radiological strategy, pharmacological agent like complex ant or other molecule with a delivery system relevant effect reducing global toxicology could be obtained" [4].

According Katritsis DG, et al "CAD is the most common underlying cause of SCD in the Western world, being responsible for 75-80 \% of cases; cardiomyopathies and genetic channelopathies account for most of the remainder. SCD accounts for $50 \%$ of all CAD-related deaths. The incidence of SCD-related atherosclerotic CAD is 0.7 per 100,000 person-years in 18-35-year-olds, increasing to 13.7 per 100,000 in those $>35$ years of age. Female survivors of cardiac arrest are less likely to have underlying CAD (45\%); valve disease and dilated or arrhythmical cardiopulmonary are more common [5]. 
According Hung MJ et al., "The causes and the mechanisms underlying the development of CAS are still poorly defined and are likely multifactorial. In the 1980s, the autonomic nervous system was found to play an important role in the pathophysiology of CAS. In the 1990 YEARS, some factors as fluorosis-inflammation, endothelial vessel dysfunction, oxidative stress agents, respiratory alkalosis and magnesium (Mg) deficiency were identified as predisposing factors. In the late 1990s and early 2000s, genetic mutations were found to be associated with CAS. Nonetheless, coronary vascular smooth muscle cell hyper-reactivity seems to constitute the substrate for CAS" [6,7]. Oros P et al., writed that "Sudden death is an important but widely under-recognized consequence of stroke. Acute stroke can disturb central autonomic control, resulting in myocardial injury, electrocardiographic abnormalities, cardiac arrhythmias, and ultimately sudden death.

Experimental-clinical objective evidence suggests that autonomic imbalance situations is more frequent after brain infarcts involving the insular cortex region, crucial for the control of autonomic functions level. (Sympathetic-parasympathetic). Cardiovascular comorbidities increase the risk of cardiac morbidity and mortality after stroke. Thus, many sudden deaths and serious non-fatal cardiac events after stroke are probably due to an interaction between cardiovascular and neurological causes. The exact mechanisms leading to sudden death remain incompletely understood. Further research is needed to investigate the autonomic consequences of acute stroke and to identify patients at high risk of sudden death" [8].

According Avila MD, et al., Results of the first large-scale randomized trial of this treatment. "TACT a large-scale clinical trial of chelation therapy for some atherosclerotic coronary pathology, found that EDTA (a chelation therapy) reduced the risk of a composite of adverse CV clinical outcomes, especially among patients with diabetes. Before disodium EDTA chelation can take its place among other accepted therapies in the routine care of post-MI patients, however, it is important that further replicative and mechanistic clinical trials be performed" [9].

Ira J Goldberg et al., writed that "Evidence that human cardiac dysfunction is associated with excess lipid, Clinical data show that both obesity and diabetes markedly increase risk of heart failure even in the absence of ischemic vascular disease.

The molecular mechanisms could be either increased the lipid uptake or an impaired mitochondrial oxidative function leading to accumulation of molecules of TGs and toxic lipid species such as ceramists, which cause myocyte loss through apoptosis, induction of iNOS and pro-hypertrophic signaling. Therefore, the specific form of excess cardiac lipid products-compounds, their cellular compartmentalization and storage form (lipid droplets), and the specific cause of CHF heart failure are likely to determine the importance of lip toxicity in human disease. Studies of human HAERT function/metabolism rely on imaging methods (relatively non-invasive).

PET scanning DIAGNOSTIC assesses the uptake of various tracers into the heart. (Well standardized for the glucose and FFA uptake). Myocardial PET imaging technique has consistently showed the increased FFA uptake and oxidation, impaired glucose uptake, in diabetic patients with normal systolic and mildly impaired diastolic function). In Recent time, RM magnetic resonance protocols have been developed to track TG metabolism such as $1 \mathrm{H}$ magnetic resonance spectroscopy (MRS).

Although hypertension and coronary artery disease are common in obese and diabetic patients, reduced heart function independent of these underlying disorders may relate to toxicities from excess metabolic substrates and defective insulin action. Some studies in patients with obesity and diabetes correlated TG accumulation with left ventricular hypertrophy. More TG has also been found in failing hearts of patients with obesity or diabetes at the time of transplantation. Reducing plasma lipids to reduce 
lipid uptake and converting oxidation to more glucose and less FA might be a method to treat patients with lip toxic and ischemic heart failure. Agents that inhibit FA oxidation have been used for angina [12]. According Mladěnka $\mathrm{P}$ et al., " $\mathrm{CV}$ diseases are a leading cause of morbidity and mortality in most developed countries of the world. DRUGS, illicit drugs, and toxins can significantly contribute to the overall cardiovascular burden.

The compounds are in this paper classified into agents that have significant effects on the heart, blood vessels, or both. The mechanism(s) of toxic action are discussed and the treatment modalities are briefly mentioned in relevant cases. Due to the large number of clinically relevant compounds and molecules discussed, this paper could be of interest to a broad audience including pharmacologists, toxicologists, clinical pharmacists, physicians, and medicinal chemists, medical laboratory professionals and other particular emphasis is given to the clinically relevant topics and interesting including the cardiovascular toxicity of illicit sympathomimetic drugs (e.g., cocaine, amphetamines, cathinones), drugs that prolong the QT interval, anti-dysrhythmia drugs, digoxin and others molecules, cardio-active steroids, beta blockers, $\mathrm{CA}++$ channel blockers, female hormones, FANS no steroidal anti-inflammatory, and anticancer compounds as anthracyclines and targeted therapy interfering with the HER2 or the vascular endothelial growth factor pathway [13].

Trang A et al., "Diabetes and heart failure PATHOLOGY commonly coexist and portend worsened prognosis than either disease alone [14]. Sharma A et al. "In this analysis of a contemporary cohort of patients with diabetes and ASCVD, sudden death was the most common subcategory of $\mathrm{CV}$ death. HF prevention may represent an avenue to reduce the risk of specific CV death subcategories" [15].

According Dziubak A, "Metformin, currently recommended as the drug of first choice in type 2 diabetes mellitus (T2DM), is one of the few anti hyperglycemic drugs to reduce cardiovascular risk. Nonetheless, due to the risk of lactic acidosis during metformin therapy, its usage in patients with diabetes and heart failure (HF) is still a matter of debate. The aim of this review is to present data supporting the possibility of using metformin in the treatment of diabetic patients with concomitant heart failure. In the failing heart, metformin through the mechanism related to AMP-activated protein kinase (AMPK) activity, improves free fatty acids (FFA) and glucose metabolism, mitochondrial biogenesis, as well as nitric oxide (NO)-NO synthase pathway. Metformin can also inhibit the generation and accumulation of advanced gyration end products (AGEs) and thereby prevents the development of the adverse structural and functional changes in myocardium. In summary, experimental and clinical data indicate the ability of metformin to prevent the development of the structural and functional changes in myocardium, although further basic research and clinical studies assessing benefits and safety of metformin therapy in patients with HF are required" [16].

According Editorial Efficacy of Oncologic Drug Therapy Some to Rethink in the Management of the System? Journal of business management and economics. JBME Luisetto M "Every medical discipline has disease treated with high or medium or low results so in the same way we can have drugs with different profile of efficacy. We can easy observe that many disease have efficacy drug therapy, often only 1 drug resolve the pathological condition. In order to have more clinical results are used association (in example one of the first used was Sulfametoxazole-trimetroprim in antimicrobial filed) But in many disease even associating 2-3-4 drugs the $\%$ of cure of a diseases not increase. What it mean? In example we can see In many oncologic disease or in metabolic disorder (as type 2 diabetes) are currently used association in order to improve clinical outcome. It mean that this drugs are not the best? Or low active? Why for this pathological condition drugs not do the right works? There is the need for new really efficacy drugs that show a profile of efficacy as requested in order to resolve the pathological condition?"[17].

Arora S, Probst MA, "A randomized, controlled trial of oral versus intravenous fluids for lowering blood glucose in emergency department patients with hyperglycemia. 
Blood glucose can be lowered via insulin and/or fluid administration. Insulin, although efficacious, can cause hypoglycemia and hypokalemia. Fluids do not cause hypoglycemia or hypokalemia, but the most effective route of fluid administration has not been well described. This study compared the efficacy and safety of oral versus intravenous fluids for reducing blood glucose in patients with hyperglycemia.

We conducted a prospective, no blinded, randomized, and controlled trial. Inclusion criteria were blood glucose $>13.9 \mathrm{mmol} / \mathrm{L}$, age $>18$ years, and ability to tolerate oral fluids. Subjects were excluded for critical illness, contraindication to fluids, and/or hyperglycemia therapy prior to enrolment. Subjects were randomized to receive oral bottled water or intravenous normal saline (maximum $2 \mathrm{~L}$ ) over 2 hours. The primary outcome of interest was a change in blood glucose at 2 hours across treatment arms.

The 48 subjects were randomized. Baseline blood glucose levels and total amount of fluid received were similar between the two groups. The mean decrease in blood glucose at 2 hours was similar for both treatment arms: a mean decrease of 3.4 $\mathrm{mmol} / \mathrm{L}(20.2 \mathrm{mmol} / \mathrm{L}$ (about $360 \mathrm{mg} / \mathrm{dl}$ to $16.8 \mathrm{mmol} / \mathrm{L}$ (about $306 \mathrm{mg} / \mathrm{dl}$ ) in the oral fluid group versus a mean decrease of $4.0 \mathrm{mmol} / \mathrm{L}(19.7 \mathrm{mmol} / \mathrm{L}$ to $15.7 \mathrm{mmol} / \mathrm{L})$ in the intravenous fluid group. The mean difference between groups was $-0.6 \mathrm{mmol} / \mathrm{L}$ (95\% confidence interval-2.3-1.2; $\mathrm{p}=0.51$ ). No adverse events were observed in either group. In this unblended randomized trial, oral and intravenous fluids were equally efficacious in lowering blood glucose levels in stable hyperglycemic patients and no adverse events were noted. Physicians should be mindful that, although similar, the reduction in blood glucose was modest in both groups" [18].

\section{Research Design and Methods}

Participants were 3,615 middle-aged men and women, with normal baseline fasting glycaemia (FG), who were recruited in a 9-year follow-up study. Odds ratios (ORs) and 95\% CIs for the incidence of hyperglycemia ( $\mathrm{FG} \geq 6.1 \mathrm{mmol} / \mathrm{L}$ or treatment for diabetes) were calculated according to daily water intake classes based on a selfadministered questionnaire. Baseline characteristics of the 3,615 norm glycemic participants are presented according to their class of W-Intake. Among them, during follow-up, 565 subjects became hyperglycemic and 202 developed diabetes. The daily $\mathrm{W}$-Intake was negatively associated with the risk of new-onset hyperglycemia, even after adjustment for multiple metabolic risk factors. Compared with daily W-Intake of $<0.5 \mathrm{~L}$, ORs were 0.64 (95\% CI 0.49-0.83) and $0.73(0.55-0.97)$ for classes of $0.5-1.0 \mathrm{~L}$ and $>1.0 \mathrm{~L}$, respectively $(\mathrm{P}=0.003)$.

The negative association of $\mathrm{W}$-Intake and risk for hyperglycemia was relevant among many subsets of participants, and those reporting a low W-Intake $(<0.5 \mathrm{~L})$ had a higher risk for hyperglycemia (for example, participants in the high physical activity group). This indicates that identification of individuals with a W-Intake of $<0.5 \mathrm{~L}$ may be widely relevant to target preventive interventions regarding the metabolic risk.

Our study has several limitations. Diabetes incidence was low and statistical power was thus limited; 24-h urine volume was not measured, but the urinary density was inversely associated with self-reported W-Intake, arguing for the validity of the questionnaire. In addition, we cannot exclude residual confounding: healthier behaviors correlating with higher water drinking could account for the observed association. Finally, only volunteers were included and the results may not be extrapolated to the general population. Our study extends this observation, drawing attention to a low $\mathrm{W}$-Intake as a possible new risk factor for impaired glycaemia. It suggests that an increase in W-Intake, an easy and costless intervention, could prevent or delay the onset of hyperglycemia and subsequent diabetes [19].

\section{Discussion}

After Observing the reported literature (In some heart disease and endocrinemetabolic conditions) in some phenomena are deeply involved: Kinetics and gradients 
in metabolism, catabolism, time related, toxic like effect, electrical cell membrane status, smooth vascular muscle cell hyper-reactivity , platelet iperactivations, central autonomic control after acute stroke, great electrolytes unbalances et other. Using and antidotes-toxicological approach, new delivery systems and new diagnostic approach in ranking the clinical risk it can be obtain new Pharmacological strategies useful in some cardiovascular conditions. To prevent some kind of heart disease and related endocrinemetabolic conditions can be useful introduce new diagnostic strategy to verify in stressing conditions the local metabolic heart performance in example in young or before high sports activities. In some cardiovascular disease as atherosclerotic pathology or diabetes type II or other metabolic-endocrine conditions can be considered as chronic endogenous poisoning? Some endogenous molecules as lipids, oxidative substances, glucose or other as activated platelet can be compared like endogenous poisons? Can be compared pro-hypertrophic signaling to an endogenous modification effect?

\section{Conclusion}

A deep knowledge in the right mechanism(s) of toxic action in some metabolicendocrine conditions using a specific toxicological approach can produce new research hypothesis to be verified for new pharmacological strategies to be introduced. Using and antidotes-toxicological approach or other depurative strategies, new delivery systems and new diagnostic approach in ranking the clinical risk new Pharmacological strategies it can be obtained useful results in some cardiovascular-metabolic endocrine conditions. If considered as an endogenous poisoning like conditions are correctly used antidotes strategies or other depurative procedure in current therapy?

\section{Clarification}

This paper has not any diagnostics or therapy intent only to produce new research hypothesis.

\section{References}

1. Luisetto M, Luca C, Farhan AK, Ghulam RM. Sudden Heart Pathology-a New Research Hipotesys in Drug Design strategy. Mod Appro Drug Des. 2017; 8: 555730. Ref.: https://goo.gl/uKLqCu

2. luisetto M, Ahmadabadi BN, Mashori GR. Heart disease new hypotesys:under endogenous toxicological aspect. J Cardiol Cardiovasc Med. 2018; 3: 001-004. Ref.: https://goo.gl/zmW7uG

3. Luisetto M. Intra- Local Toxicology Aspect Time Related in Some Pathologic Conditions. Open Acc J of Toxicol. 2017; 2: 002 555586. Ref.: https://goo.gl/ghchpJ

4. Luisetto M, Nili-Ahmadabadi Bm, Mashori GR. Surgery and new Pharmacological strategy in some atherosclerotic chronic and acute conditions. Arch Surg Clin Res. 2017; 1: 042-048. Ref.: https://goo.gl/xYWA29

5. Katritsis DG, Gersh BJ, Camm AJ. A Clinical Perspective on Sudden Cardiac Death. Arrhythm Electrophysiol Rev. 2016; 5: 177-182. Ref.: https://goo.gl/xoti5Z

6. Hung MJ, Hu P, Hung MY. Coronary artery spasm: review and update. Into J Med Sci. 2014; $11: 1161$ 1171. Ref.: https://goo.gl/hc7ZvS

7. Myerburg RJ, Junttila MJ. Sudden cardiac death caused by coronary heart disease. Circulation. 2012; 125: 1043-1052. Ref.: https://goo.gl/bf2HZa

8. Sörös $P$, Hachinski V. Cardiovascular and neurological causes of sudden death after ischemic stroke. Lancet Neurol. 2012; 11:179-188. Ref.: https://goo.gl/5xrqMg

9. Avila MD, Escolar E, Lamas GA. Chelation therapy after the Trial to Assess Chelation Therapy: results of a unique trial. Curr Opin Cardiol. 2014; 29: 481-488. Ref.: https://goo.gl/Vk9bkr

10. Buxton AE. Sudden death in ischemic heart disease-2017. Int JCardiol. 2017; 237: 64-66. Ref.: https://goo.gl/7FvKnS

11. Shen L, Jhund PS, Petrie MC, Claggett BL, Barlera S, et al. Declining Risk of Sudden Death in Heart Failure. N Engl J Med. 2017; 377: 41-51. Ref.: https://goo.gl/tgokvo

12. Goldberg IJ, Trent CM, Schulze PC. Lipid Metabolism and Toxicity in the Heart. Cell Metab. 2012; 15 805-812. Ref.: https://goo.gl/3vbe6d 
13. Mladěnka $P$, Applová L, Patočka J, Costa VM, Remiao F, et al. Comprehensive review of cardiovascular toxicity of drugs and related agents. Med Res Rev. 2018. Ref.: https://goo.gl/282h1J

14. Trang A, Aguilar D. Treating Disease Mechanisms in Patients with Heart Failure and Diabetes Mellitus. Curr Heart Fail Rep. 2017; 14: 445-453. Ref.: https://goo.gl/xGt3U3

15. Sharma A, Green JB, Dunning A, Lokhnygina Y, Al-Khatib SM, et al. Causes of Death in a Contemporary Cohort of Patients With Type 2 Diabetes and Atherosclerotic Cardiovascular Disease: Insights From the TECOS Trial. Diabetes Care. 2017; 40: 1763-1770. Ref.: https://goo.gl/LjdQeR

16. Dziubak A, Wójcicka G. The pathophysiological basis of the protective effects of metformin in heart failure. Postepy Hig Med Dosw (Online). 2017; 71: 773-787. Ref.: https://goo.gl/r3oekn

17. Editorial Efficacy of Oncologic Drug Therapy Some to Rethink in the Management of the System? Journal of business management and economics. JBME luisetto $m$

18. Arora S, Probst MA, Andrews L, Camilion M, Grock A, et al. A randomized, controlled trial of oral versus intravenous fluids for lowering blood glucose in emergency department patients with hyperglycemia. CJEM. 2014; 16: 214-219. Ref.: https://goo.gl/7A3XEo

19. Roussel R1, Fezeu L, Bouby N, Balkau B, Lantieri O, et al. Low Water Intake and Risk for New-Onset Hyperglycemia. Diabetes Care. 2011; 34: 2551-2554. Ref.: https://goo.gl/LCiw1q 